\title{
Primary Prophylaxis of Gastric Variceal Bleeding Comparing Cyanoacrylate Injection and Beta-Blockers in Patients with Liver Cirrhosis: A Randomized Controlled Trial
}

\author{
Salama A G, M A Tawfik*, Farrag W and Harras F \\ Internal Medicine Department, Faculty of Medicine, Tanta University, Egypt
}

Submission: August 18, 2020; Published: September 01, 2020

*Corresponding author: Mohamed A Tawfik, Department of Internal Medicine, Faculty of Medicine, Tanta University, Tanta, Egypt

Abstract

Background and study aim: The bleeding from gastric varices are more severe, requiring more blood transfusions, and has a higher mortality rate than esophageal variceal bleeding, there is little data on primary prophylaxis of GV bleeding, we compared the efficacy of cyanoacrylate injection and beta-blockers in primary prophylaxis of gastric variceal bleeding.

Methods: Cirrhotics with gastroesophageal varices type 2 with eradicated esophageal varices or large isolated gastric varix type 1, who had never bled from gastric varix, were randomized to cyanoacrylate injection (Group I, $n=30$ ), beta-blockers (Group II, $n=30$ ) or no treatment (Group III, $n=30$ ). Primary endpoints were bleeding from gastric varix or death.

Results: bleeding from gastric varices over 6 months follow up was 6.67\% in Group I, 20\% in Group II, 36.67\% in Group III (p = 0.017). GV obliterated or decreased in size $42.86 \%$ and $57.14 \%$ of group I respectively. No patients neither in group II nor in group III had an obliteration or decrease in GV size. $(\mathrm{p}<0.001)$.

Conclusion: Primary prophylaxis in patients with gastric varices is recommended to reduce the risk of first bleeding. Cyanoacrylate injection is more effective than beta-blocker therapy in preventing first gastric variceal bleeding.

Keywords: Cirrhosis; Endoscopy; Gastric varices; Cyanoacrylate

\section{Introduction}

Gastric varices (GV) are present in around $20 \%$ of patients with cirrhosis, portal hypertension and varices detected in the endoscopy [1].The risk of bleeding is lower than with esophageal varices, yet the transfusion requirements and mortality associated with a bleeding episode are both higher. Risk factors for GV bleeding are similar to those of esophageal varices and include size of fundal varices, child's class, and red spots [2]. The 2-year bleeding risk for larger gastric varices can be as much as $65 \%[2,3]$. GV are more common in segmental portal hypertension caused by portal/splenic vein thrombosis, than in generalized portal hypertension due to cirrhosis [4]. GV are classified According with their anatomical site, size or their development before or after endoscopic/surgical intervention. According to the site there are 4 types, Gastroesophageal varices (GOV)-1 and 2; are OV extending beneath the cardia through the lesser curvature and fundus respectively, Isolated gastric varices
(IGV)-1 and 2; are not connected with OV and they are located on the fundus only and stomach only respectively. According to their size, they are classified in small $(<5 \mathrm{~mm})$, medium (5 to 10 $\mathrm{mm}$ ) and large ( $>10 \mathrm{~mm}$ ). According to their development before or after endoscopic/surgical intervention Primary GV are present at the time of the initial endoscopy and before any endoscopic/ surgical intervention, and secondary GV are those which appear after endoscopic/surgical intervention [5]. The 2-year incidence of variceal bleeding from IGV1 and GOV2 type of varices is more frequent and profuse (78\% and $54 \%$, respectively) than the lesser curve (GOV1) varices (28\%) [1]. There is not enough evidence on secondary prophylaxis and even less on primary prophylaxis to make strong recommendations. Probably, cyanoacrylate injection has a role in both primary and secondary prophylaxis, but most experts prefer to suggest NSBB in primary prophylaxis as they are less invasive and easily accessible. There is still a wide area 
for research in GV therapy [5]. We performed a randomized controlled study to compare the efficacy of endoscopic cyanoacrylate injection versus beta blockers, versus no treatment in the prevention of GV bleeding.

\section{Patients and Methods}

This randomized controlled study was performed at the Gastroenterology Unit of Internal Medicine Department at Tanta University Hospitals. Ninety patients proved to have liver cirrhosis were included in this study in the period from May 2013 to December 2017. Patients were randomized in 1:1:1 ratio using sealed envelope technique to receive endoscopic cyanoacrylate injection (Group I) or beta-blocker (Group II) or no treatment (Group III). The random allocation sequence remained concealed from the investigators until the intervention was assigned. Endoscopic and beta blocker treatments were started immediately after randomization. The follow up was started at the time of randomization and was done for at least 6 months.

\section{Inclusion criteria}

All included patients were diagnosed by ultrasound to have liver cirrhosis, portal hypertension complicated by GV in upper gastrointestinal endoscopy. Primary small, medium and large GVs, types IGV1 and GOV2 with no evidence of gastric variceal bleeding and eradicated esophageal varices.

\section{Exclusion criteria}

Non eradicated Esophageal varices, Secondary type GV, Portal vein thrombosis, non-cirrhotic portal hypertension, Hepatocellular Carcinoma, Child Pugh (C) cirrhosis, major cardiac or chest comorbidities, diabetes meletus and those who refused to give informed consent. The size of GV was calculated by zebra guide wire with markings made at $5 \mathrm{~mm}$ interval and the fundal varix was classified as small $(<10 \mathrm{~mm})$ and large $(>10 \mathrm{~mm})$. The color of GV was observed as red or blue. The presence or absence of EV and the portal hypertensive gastropathy were also evaluated. An upper gastrointestinal endoscopy was done to determine the variceal status at baseline and was repeated at 6-month intervals or during bleeding. Size of GV, appearance of EV, and portal hypertensive gastropathy were recorded. Conscious sedation using midazolam was used prior to the endoscopic procedure. Variceal bleeding was defined as occurrence of hematemesis and/ or melena requiring P2 $\mathrm{U}$ of blood or a decrease of $3 \mathrm{gm} / \mathrm{dl}$ of hemoglobin if no blood transfusion is given [6].

\section{Definition of haemorrhage from GV}

The evidence of gastric variceal bleeding included (A) active spurting or oozing of blood from gastric varices during endoscopy and (B) stigmata of recent hemorrhage such as blood clots coating on the gastric varices or ulcer on gastric varices. Primary endpoints of the study were bleeding from GV or death. The actuarial probabilities of bleeding from GV and mortality were calculated. Secondary endpoints were increase or decrease in the size of GV, appearance of new EV, and appearance or worsening of portal hypertensive gastropathy and complications. The diagnosis of cirrhosis was based on clinical, biochemical, radiological, and histological findings. Ultrasonography of the abdomen. ChildTurcotte-Pugh scores were calculated at baseline and serial intervals. Patients were advised to refrain from consuming alcohol and taking non-steroidal anti-inflammatory drugs.

\section{Technique of cyanoacrylate injection}

Standardized cyanoacrylate injection technique: N-butyl2-cyanoacrylate ampoule $(0.5 \mathrm{~mL})$ (GluStitch ${ }^{\circledR}$ Twist, GluStitch Inc. Delta, BC, Canada) diluted with $0.8 \mathrm{~mL}$ of lipiodol ${ }^{\circledR}$ was injected using Pentax video-endoscopy and a 23-gauge disposable injection needle (Wilson-Cook Medical Inc., USA), immediately followed by 1- $2 \mathrm{ml}$ distilled water to flush out the remaining cyanoacrylate from the dead space of the catheter into the injected varix then the needle was retracted.

An attempt was made to completely obturate the GV in one session, by injecting cyanoacrylate at multiple sites. If a second session was considered necessary, it was done within one week of the initial session. The injected GV was palpated using the hub of the injector with the needle retracted to determine solidification and obliteration of the GV. If the GV was not completely obturated, cyanoacrylate was reinjected until all the GV became solidified. Caution was taken to prevent damage to endoscope by applying silicon oil over the endoscope tip and flushing the endoscope channel with acetone. All patients in the cyanoacrylate group received proton pump inhibitors for 4 weeks [7].

\section{Beta-blockers therapy}

Propranolol was started at a dose of $20 \mathrm{mg}$, twice daily. The dose was increased by $20 \mathrm{mg}$ every alternate day to achieve a target heart rate of $55 / \mathrm{min}$ or to decrease the heart rate by $25 \%$ from baseline [8]. Patients were informed about the purpose and procedure of the study and benefits of sharing in it. The study was approved by the Hospital Ethical Committee and was performed according to the Principles of the Declaration of Helsinki, a written informed consent of the patients was obtained. The studied groups were age and sex matched.

\section{Statistical analysis of the data}

First, we calculated the sample size between cyanoacrylate injection group and each of beta-blocker no-treatment group and found that 30 patients will be required in each group, to achieve a Confidence Level of $90 \%$ with a margin of error of $15 \%$. Fisher's exact test was used to compare categorical groups. Student's t-test and Mann-Whitney U-test were used for the parametric and non-parametric data, respectively. One-way ANOVA test was used to compare baseline parameters and treatment endpoints. For comparison of pre- and post-therapy parameters between groups, the paired Student's t-test was used. Each patient was analyzed in an intention to treat manner. $p$ Value of $<0.05$ was considered significant. loss of follow-up or end of the study ( 6 months). Twosided $p$ values $<0.05$ were considered as statistically significant. 
The IBM SPSS 22.0 statistic software (SPSS Inc., Armonk, NY, USA) was used for all statistical analysis.

\section{Results}

Between May 2013 to December 2017, of 720 cirrhotic patients with portal hypertension presented to our hospital, 630 did not meet inclusion criteria and were excluded. Ninety cirrhotic patients with GV (GOV2/IGV1) without EV, who had no history of GV bleeding, were randomized to receive cyanoacrylate injection (Group I, $\mathrm{n}=30$ ), beta-blockers (Group II, $\mathrm{n}=30$ ) or no treatment (Group III, $\mathrm{n}=30$ ). The baseline characteristics of the three groups were comparable (Table 1). The most common etiology of cirrhosis was hepatitis c virus. Size of GV and portal hypertensive gastropathy in the 3 groups were similar. (Table 1 ).

Table 1: Baseline characteristics of patients.

\begin{tabular}{|c|c|c|c|c|}
\hline Parameters & Cyanoacrylate & Beta-blocker & No treatment & $p$ value \\
\hline & Group I & Group II & Group III & \\
\hline & $(n=30)$ & $(n=30)$ & $(n=30)$ & \\
\hline Age (Mean) yrs. & $55.933 \pm 9.355$ & $55.967 \pm 9.080$ & $56.233 \pm 7.569$ & 0.989 \\
\hline Sex (Male: Female) & $13: 17$ & $12: 18$ & $10: 20$ & 0.721 \\
\hline Location (rural/ Urban) & $26: 04: 00$ & 27:03:00 & $25: 05: 00$ & 0.747 \\
\hline Etiology of Cirrhosis (HCV/HBV/others) & $25 / 1 / 4$ & $22 / 2 / 6$ & $27 / 0 / 3$ & 0.4643 \\
\hline $\mathrm{Hb}($ mean g/dl) & $10.223 \pm 0.743$ & $10.307 \pm 0.780$ & $9.943 \pm 0.732$ & 0.152 \\
\hline Platelets (plt/mcl) & $106.050 \pm 29.655$ & $106.333 \pm 24.466$ & $93.150 \pm 16.640$ & 0.06 \\
\hline White blood cells (wbcs/mcl) & $5663.33 \pm 2045.092$ & $5673.33 \pm 1715.836$ & $5310.0 \pm 1564.333$ & 0.67 \\
\hline $\operatorname{ALT}(\mathrm{u} / \mathrm{L})$ & $66 \pm 16.034$ & $67.5 \pm 15.206$ & $64 \pm 15.205$ & 0.774 \\
\hline $\operatorname{AST}(\mathrm{u} / \mathrm{L})$ & $58.2 \pm 13.71$ & $58.067 \pm 14.377$ & $55.7 \pm 13.797$ & 0.738 \\
\hline Serum Albumin (g/dl) & $3.633 \pm 0.370$ & $3.653 \pm 0.298$ & $3.503 \pm 0.258$ & 0.136 \\
\hline Prothrombin time (seconds) & $14.567 \pm 0.971$ & $14.133 \pm 0.776$ & $14.3 \pm 0.794$ & 0.145 \\
\hline Bilirubin (mg/dl) & $1.837 \pm 0.522$ & $1.557 \pm 0.532$ & $1.807 \pm 0.603$ & 0.105 \\
\hline Urea (mg/dl) & $33.267 \pm 8.043$ & $32.2 \pm 8.385$ & $32.733 \pm 7.597$ & 0.876 \\
\hline Creatinine $(\mathrm{mg} / \mathrm{dl})$ & $0.69 \pm 0.359$ & $0.703 \pm 0.393$ & $0.657 \pm 0.364$ & 0.883 \\
\hline Ascites (no/yes) & $23 / 7$ & $24 / 6$ & $22 / 8$ & 0.83 \\
\hline Portal Hypertensive Gastropathy & 7 & 9 & 9 & 0.211 \\
\hline Size of GV (small/moderate/large) & $23 / 5 / 2$ & $27 / 3 / 0$ & $29 / 1 / 0$ & 0.747 \\
\hline
\end{tabular}

Regarding, GV bleeding and over a follow-up period of 62 out of 30 (6.67\%) patients in Group I The difference in bleeding months for each patient, 6 out of 30 (20\%) patients in Group II, from GVs was significant between Group I, II and III (p = 0.017). and 11 out of $30(36.67 \%)$ patients in Group III bled, compared to $\quad$ (Table 2)

Table 2: Bleeding from GV after 6 months of follow up.

\begin{tabular}{|c|c|c|c|c|c|c|c|c|c|c|}
\hline & \multicolumn{8}{|c|}{ Groups } & \multirow{4}{*}{\multicolumn{2}{|c|}{ Chi-Square }} \\
\hline & \multirow{2}{*}{\multicolumn{2}{|c|}{$\begin{array}{c}\text { Cyanoacrylate } \\
\text { Group I }\end{array}$}} & \multirow{2}{*}{\multicolumn{2}{|c|}{$\begin{array}{c}\text { Beta-blocker } \\
\text { Group II }\end{array}$}} & \multicolumn{2}{|c|}{ No treatment } & \multicolumn{2}{|c|}{ Total } & & \\
\hline & & & & & & III & & & & \\
\hline & & & & & & & & & & \\
\hline & n & $\%$ & $\mathbf{n}$ & $\%$ & $\mathbf{n}$ & $\%$ & $\mathbf{n}$ & $\%$ & $\mathrm{X}^{2}$ & $P$ value \\
\hline No bleeding & 28 & 93.3 & 24 & 80 & 19 & 63.33 & 71 & 78.89 & & \\
\hline Bleeding GV & 2 & 6.67 & 6 & 20 & 11 & 36.67 & 19 & 21.11 & & \\
\hline Total & 30 & 100 & 30 & 100 & 30 & 100 & 90 & 100 & 8.139 & $0.017^{*}$ \\
\hline
\end{tabular}


After 6 months of follow up, endoscopic review of GVs in nonbleeding patients revealed that it was obliterated only in 12 out of 28 patients (42.86\%) of group I. Moreover, GVs were decreased in size in 16 out of 28 patients ( $57.14 \%$ ) of group I. no patient either in group II nor in group III had an obliteration or decrease in GV size. On the other side, GVs increased in size in both group II (16 out of 24$)(66.67 \%)$ and group III (19 out of 19) (100\%) but not in group I. No change in GVs size were observed only in 8 out of 24 patients (33.33\%) of group II but not in group I or III. significant difference in these results with a $(\mathrm{p}<0.001)$ (Table 3$)$.

Table 3: Results of treatment of GV after 6 months after exclusion of bleeders

\begin{tabular}{|c|c|c|c|c|c|c|c|c|c|c|}
\hline & \multirow{2}{*}{\multicolumn{2}{|c|}{$\begin{array}{c}\text { Cyanoacrylate } \\
\text { Group I }\end{array}$}} & \multirow{2}{*}{\multicolumn{2}{|c|}{$\frac{\text { Beta-blocker }}{\text { Group II }}$}} & \multirow{2}{*}{\multicolumn{2}{|c|}{$\begin{array}{c}\text { No treatment } \\
\text { Group III }\end{array}$}} & \multirow{2}{*}{\multicolumn{2}{|c|}{ Total }} & \multirow{2}{*}{\multicolumn{2}{|c|}{ Chi-Square }} \\
\hline & & & & & & & & & & \\
\hline & $\mathrm{n}$ & $\%$ & $\mathrm{n}$ & $\%$ & $\mathrm{n}$ & $\%$ & $\mathrm{n}$ & $\%$ & $X^{2}$ & P-Value \\
\hline GV Obliteration & 12 & 42.86 & 0 & 0 & 0 & 0 & 12 & 16.9 & & \\
\hline GV decrease in size & 16 & 57.14 & 0 & 0 & 0 & 0 & 16 & 22.53 & & \\
\hline GV Increase in size & 0 & 0 & 16 & 66.67 & 19 & 100 & 35 & 49.3 & 114.816 & $<0.001^{*}$ \\
\hline GV no change in size & 0 & 0 & 8 & 33.33 & 0 & 0 & 8 & 11.27 & & \\
\hline
\end{tabular}

All patients tolerated the study with no serious complications or mortality during the 6 months of follow up for each patient. No Bleeding-related mortality was happened as all patients bled were shifted to primary end point of the study and underwent an emergency endoscopy with successful hemostasis [9].

\section{Discussion}

Long time ago, there is a rising interest in the evaluation of the importance of the primary prophylaxis of the GVs in chronic liver disease patients. The overall incidence of gastric varices in patients with portal hypertension is $18 \%-70 \%{ }^{(1,9)} \mathrm{GVs}$ has the characteristics of more severe blood loss and higher mortality and is a more difficult management problem than esophageal variceal hemorrhage [10].

Egypt is known to have a wide spread of HCV infection with a portal hypertension complication, GVs bleeding represent a serious daily problem in our country specially in the region of this study at Tanta university hospital. Therefore, it is important to define successful approaches to prevent the first bleeding from GV.

The results of this prospective randomized controlled clinical trial, comparing the efficacy of beta-blockers therapy with endoscopic cyanoacrylate injection or no treatment in different sized GV, clearly show that endoscopic injection of cyanoacrylate, is significantly more effective than beta-blockers or no treatment in the prevention of first bleeding from high risk GV. These results similar to a few number of a previous studies [10-13], Some experts recommend using non-selective beta-blockers (NSBB) as primary prophylaxis and avoiding cyanoacrylate injections because they consider these studies very particular since it was conducted in a single expert centers and thus, it is considered not enough evidence to generalize its findings $[14,15]$. However, our results in this study support the same direction.

Objecting opinions see that glue injection requires expertise which is not always available and the low complication rate in these studies reflects the high skills of endoscopists who conducted it, which is not widely reproducible. Moreover, they see that the rebleeding rate remains high, at $22 \%-34 \%$ and episodic complications such as systemic embolism have been the main issues [16]. Cerebral stroke, portal vein embolization, splenic infarction, coronary embolism, and nonfatal pulmonary emboli in $4.6 \%$ of cases were reported as complications of tissue adhesive use [17-20]. But the complication rate was relatively low and most of the complications were not severe. The common adverse effects of cyanoacrylate injection therapy include fever and abdominal discomfort. In this study, no unusual adverse effects were noted in our study. Moreover, we have to remember that endoscopic injection of cyanoacrylate is the currently recommended in recent consensus and guidelines as the initial treatment for acute gastric variceal bleeding [21,22].

Data suggesting that carvedilol is more effective in reducing the hepatic venous pressure gradient (HVPG) may reduce the 
gap in efficacy between NSBB and cyanoacrylate injection. On the other hand, it is well known that bleeding from GV does not depend only on the HVPG, but also on the wall tension and size of the varix [6]. Use of $\beta$-blockers for the primary prevention of gastric variceal bleeding is not supported by significant data [23]. Because the mortality rate of gastric variceal bleeding is high, it has been suggested that patients at high risk for bleeding should undergo primary prophylaxis of the gastric varix [2,24].

In this study, GV bleeding over a follow-up period of 6 months for each patient, 6 out of $30(20 \%)$ patients in Group II, and 11 out of $30(36.67 \%)$ patients in Group III bled, compared to 2 out of 30 (6.67\%) patients in Group I. The difference in bleeding from GVs was significant between Group I, II and III ( $\mathrm{p}=0.017)$. in Mishra et al. rebleeding was $10 \%, 38 \%$ and $53 \%$ for groups I, II and III respectively [10]. while in Chang YJ et al, study no bleeding occurs in $76.92 \%$ in those who received cyanoacrylate injection [12].

After 6 months of follow up, endoscopic review of GVs in nonbleeding patients revealed that it was obliterated only in 12 out of 28 patients (42.86\%) of group I. Moreover, GVs were decreased in size in 16 out of 28 patients (57.14\%) of group I. no patient either in group II nor in group III had an obliteration or decrease in GV size. These results were very close to the previous studies [10-13].

No mortality were observed in the 3 groups in this study which can be explained by the short period of follow up for each patient (6 months) , the emergency endoscopy which was made for all bleeders after resuscitation and lastly because we excluded child Pugh C patients as they are at risk of death not only from bleeding but from liver cell failure, hepatorenal syndrome, hepatocellular carcinoma and more.

\section{Conclusion}

In conclusion, Primary prophylaxis in patients with gastric varices is recommended to reduce the risk of first bleeding. Cyanoacrylate injection is more effective than beta-blocker therapy in preventing first gastric variceal bleeding.

\section{References}

1. Sarin SK, Lahoti D, Saxena SP, Murthy NS, Makwana UK (1992) Prevalence, classification and natural history of gastric varices: a longterm follow-up study in 568 portal hypertension patients. Hepatology 16(6): 1343-1349.

2. Kim T, Shijo H, Kokawa H, Tokumitsu H, Kubara K, et al. (1997) Risk factors for hemorrhage from gastric fundal varices. Hepatology 25(2): 307-312.

3. Shiba M, Higuchi K, Nakamura K, Itani A, Kuga T, et al. (2002) Efficacy and safety of balloon occluded endoscopic injection sclerotherapy as a prophylactic treatment for high-risk gastric fundal varices: a prospective, randomized, comparative clinical trial. Gastrointest Endosc. 56(4): 522-528.

4. Madsen MS, Petersen TH, Sommer H (1986) Segmental portal hypertension. Ann Surg 204(1): 72-77.

5. Vine LJ, Subhani M, Acevedo JG (2019). Update on management of gastric varices. World J Hepatol 11(3): 250-260.
6. de Franchis R (2005). Evolving Consensus in Portal Hypertension Report of the Baveno IV Consensus Workshop on methodology of diagnosis and therapy in portal hypertension. J Hepatol 43(1): 167176.

7. Elsebaey MA, Tawfik MA, Ezzat S, Selim A, Elashry H, et al. (2019) Endoscopic injection sclerotherapy versus N-Butyl-2 Cyanoacrylate injection in the management of actively bleeding esophageal varices: a randomized controlled trial. BMC Gastroenterol 19(1): 23.

8. Mohamed A Tawfik, Abd Allah Ahmed El-Sawy (2016) Endoscopic Band Ligation Versus Propranolol in Primary Prophylaxis of Esophageal Variceal Bleeding and Improvement of Survival in Hepatocellular Carcinoma Patients. JMPS 6(1): 47-58.

9. Watanabe K, Kimura K, Matsutani S, Ohto M, Okuda K (1988) Portal hemodynamics in patients with gastric varices. A study in 230 patients with esophageal and/or gastric varices using portal vein catheterization. Gastroenterology 95(2): 434-440.

10. Mishra SR, Sharma BC, Kumar A, Sarin SK (2011) Primary prophylaxis of gastric variceal bleeding comparing cyanoacrylate injection and beta-blockers: A randomized controlled trial. J Hepatology 54(6): 1161-1167.

11. Tripathi D (2011) Primary prophylaxis against gastric variceal bleeding: is there a sticky solution at last? Hepatology 54(3): 10941096.

12. Kang EJ, Jeong SW, Jang JY, Cho YJ, Lee HS, et al. (2011) Long-term result of endoscopic Histoacryl (N-butyl-2-cyanoacrylate) injection for treatment of gastric varices. World J Gastroenterol 17(11): 1494-1500.

13. Chang Y J, Park J J, Joo M K, Lee B J, Yun J W, et al. (2010) Long-Term Outcomes of Prophylactic Endoscopic Histoacryl Injection for Gastric Varices with a High Risk of Bleeding. Dig Dis Sci 55(8): 2391-2397.

14. Garcia-Tsao G, Abraldes J G, Berzigotti A, Bosch J (2017) Portal hypertensive bleeding in cirrhosis: Risk stratification, diagnosis, and management: 2016 practice guidance by the American Association for the study of liver diseases. Hepatology 65(1): 310-335.

15. Tripathi D, Stanley A J, Hayes P C, Patch D, Millson C, et al. (2015) Clinical Services and Standards Committee of the British Society of Gastroenterology. U.K. guidelines on the management of variceal haemorrhage in cirrhotic patients. Gut 64(11): 1680-1704.

16. Naga M, Foda A (1997) An unusual complication of histoacryl injection. Endoscopy 29: 140.

17. Yu L K, Hsu C W, Tseng J H, Liu N J, Sheen I S (2005) Splenic infarction complicated by splenic artery occlusion after N-butyl-2-cyanoacrylate injection for gastric varices: case report. Gastrointest Endosc 61(2): 343-345.

18. Roesch W, Rexroth G (1998) Pulmonary, cerebral and coronary emboli during bucrylate injection of bleeding fundic varices. Endoscopy 30(8): S89-S90.

19. Hwang S S, Kim H H, Park S H, Kim S E, Jung J I, et al. (2001) N-butyl2-cyanoacrylate pulmonary embolism after endoscopic injection sclerotherapy for gastric variceal bleeding. J Comput Assist Tomogr 25(1): 16-22.

20. Palejwala A A, Smart H L, Hughes M (2000) Multiple pulmonary glue emboli following gastric variceal obliteration. Endoscopy 32(1): S1-S2.

21. Laine L, Planas R, Nevens F, Banares R, Patch D (2006) Treatment of the acute bleeding episode. In: Franchis R (Edt.), Consensus Workshop on Definitions, Methodology and Therapeutic Strategies. Oxford: Blackwell Science, pp. 217-242.

22. Garcia-Tsao G, Sanyal A J, Grace ND, Carey W (2007) Prevention and management of gastroesophageal varices and variceal hemorrhage in cirrhosis. Hepatology 46(3): 922-938. 
23. Ryan B M, Stockbrugger R W, Ryan J M (2004) A pathophysiologic, gastroenterologic, and radiologic approach to the management of gastric varices. Gastroenterology 126(4): 1175-1189.
24. Matsumoto A, Matsumoto H, Hamamoto N, Kayazawa M (2001) Management of gastric fundal varices associated with a gastrorenal shunt. Gut 48(3): 440-441.

This work is licensed under Creative Commons Attribution 4.0 License DOI:10.19080/ARGH.2020.15.555922 